\title{
Research on Interior Design Education Based on Narrative Theory
}

\author{
Yongxin $\mathrm{Wu}$ \\ School of Design,Jianghan University,Wuhan,430056,P.R.CHINA \\ 82416631@163.com
}

\begin{abstract}
Keywords: Narratives Theory, Interior Space, Interior Design Education
\end{abstract}
\begin{abstract}
Through narrative theory use in interior design, interior design also illustrate the narrative, the diversity of interior design is unique narrative interior narrative resources, indoor narrative space theory, space mining unique narrative resources, and on this basis restore lost event indoor space, create interior space, Establish the cultural connotation of interior space; while the use of text analysis and map overlay method, to identify unique narrative belongs to interior space, which will benefit the cultural construction of indoor and culture protection. Interior design narrative, the academic level of urban development, which can take on the burden of the cultural heritage of the city; the technical level of urban development and construction, it can be used as an analytical tool for urban culture, an important article proposed by Interior Design theory accumulated achievements in narrative research methods, and through the specific facts of the case study analysis of narrative theory results and interior design practice of effective link and transformation in order to establish the theoretical value of this study.
\end{abstract}

\section{Introduction of Narrative Theory}

The so-called narrative is the use of a specific language to express a story. Simply put, it is to tell stories, with storytelling events narrated want to express, but in the creative process through a variety of Syrian behavior in terms of how good a story of how a simple event in chronological order, edited into an organized plot is narrative to study content.

Said elements of narrative can be summarized into two parts: the narrative content and narrative approach. Content refers to the narrative description of what and how narrative; narrative content can be used a lot, but it must be a time factor so that the entire incident varied themes. Narrative approach is varied; narrative approach has two aspects: structural representation of language and expression process. Expressed in language is all that can convey information carrier. "Conveying information carrier" choice and "expression vector information" ideographic function is critical, which determines the accuracy of the representation language. Expression of the structure is a direct impact on the effect of the expression of the major factors that determine the structure of the different expression of the different expressions of interest ${ }^{[1]}$.

Narrative continue to expand the scope of the study, research, and with a multi-disciplinary, so that a very wide range of applications. Narrative literary narrative gradually rise to social science, structure narrative, feminist narrative, Narrative movies, etc; with the rise of modern design theory, the current foreign Narrative applied to the design field, especially in architecture subject urban planning, landscape design, a detailed narrative design approach to the development of these disciplines play an effective role in guiding our research and science applied to the design in the field of narrative, not comprehensive and complete, also the need for further review and improvement.

\section{Characteristic of Interior Design Education}

Interior design is based on the nature of the use of the building, the environment and the corresponding standards, the use of material and technical means and architectural design principles to create a functional and reasonable, comfortable and beautiful, meet the people's material and spiritual life needs of the indoor environment. The space environment use value, corresponding to meet the functional requirements, but also reflects the historical context, architectural style, 
atmosphere and other psychological factors. Interior design evolved from the architectural design of the decorative parts out. Interior design of public buildings can be divided into two categories of space and home. Interior design refers to actually be able to establish any interior related items: including: walls, windows, curtains, gates, surface treatment, materials, lighting, air conditioning, water and electricity, environmental control systems, audio-visual equipment, furniture and decorations planning ${ }^{[2]}$. Fig.1 shows the characteristic of interior design education.

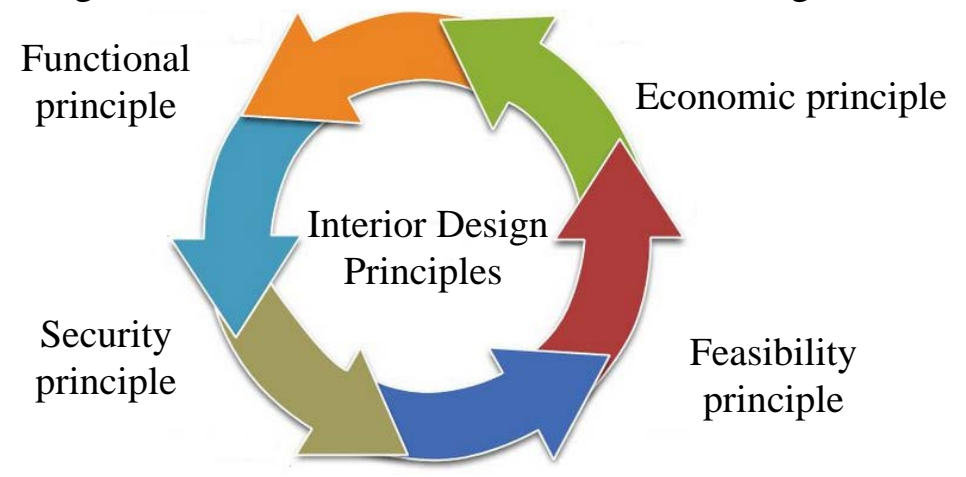

Fig. 1 The characteristic of interior design education

Functional principle: include meeting and ensure the use of the claim, to protect against damage to the main structure of the building and the facade, interior decoration, etc. Security principle: whether wall, floor or ceiling, and its structure are required to have a certain strength and stiffness, calculated in line with the requirements, particularly the connection node between the parts, but also safe and reliable. Feasibility principle: The reason for the design, construction through to the design into reality, therefore, the interior design must be feasible, and strive to construction convenient, easy to operate. Economic Principles: the actual nature of the building is different and uses design standards, do not blindly raise standards, the simple pursuit of artistic effect, resulting in a waste of money, do not be one-sided lower the standard to affect results, it is important at the same cost, by cleverly constructed to achieve a good practical design and artistic effect.

With the development of the professional community to further improve this situation will gradually improve, it will appear the following trends: interior design must return naturalization: With the growth of environmental awareness, people longing for nature, natural drink beverages, natural material, eager to live in a natural green environment. A high nationalization degree only emphasized the highly modernized, although people improve the quality of life, but the tradition was lost, lost in the past. Therefore, the development trend of modern interior design is modern-talk, and talk about tradition. Interior design for an ultra-modern: With the development of science and technology, interior designers to learn to use all the means of modern technology, so that the interior design to achieve the best sound, light, color, shape matching effect, to achieve high speed, high efficiency, high performance, to create the ideal space worthy of praise environment ${ }^{[3]}$.

\section{Museum Interior Design Principles Based on Narratives Theory}

Design museum space has many associations. Narrative is the first major study of storytelling, theoretical category rules, while the museum's space design is inseparable from the story, the story can give the museum space deep cultural and historical connotations, is the interpretation of the story of the museum space the viewer a key; at the same time the space environment can promote the development of the story, to make the museum more space contagious. Therefore, the study of narrative in the museum space design has practical significance. Narrative elements museum space design refers to the design process in the museum space to convey the whole story indispensable several factors. Designers are the narrator in the museum space design, and play a role in conveying information space design; the recipient is the viewer to visit space. Thus, the narrative elements that constitute the museum space design are designer, space, user three elements ${ }^{[4]}$.

In the museum space design elements of the narrative process is to determine the user truly, with the user to make the museum space designed to have a purpose and direction, in order to 
promote the development of spatial narrative. Through narrative designer space design, you can establish a close interaction between space and human behavior and feelings, this space may become intrinsic value carrier. Designers in the museum space design, space, the user is to convey the whole story of the essential several factors.

Currently the museum space design borrows only literary narrative structure described in the order of Syria along the way, we can also demand study design museum space borrowed flashback, interleaved and annotated supplement practices. Such as how to borrow just the right flashback, through space create suspense plot arrangement, to enhance the attractiveness of the story; how to borrow just the right way interleaved, spatial heterogeneity in a scene inserted into a series of homogeneous event scenario, space will organize a number of relatively active, with the order in the disorder; how to borrow annotated supplement just the right way, at the end of the sequence space in the museum is set to emphasize the theme, emphasizing sequence integrity and at the end of efforts to make the museum more space contagious.

\section{Museum Interior Design Case}

Narrative perspective museum space design is mainly used to express the whole perspective focusing grand historical scenes. As the designer is not set fixed viewing position, the viewer can be at any position of the corridor overlooking the panoramic viewing space in the past, present and future things happen. Omniscient view of expression allows the viewer to quickly understand the status of the entire history, but there is a lack of space for the viewer to imagine the lack of perspective and focus type inner focus type outside the perspective of expression, so that the lack of a kind of intimacy and realism. Therefore, in the museum space design can be used in the focus type perspective, show the character's mental activities through microscopic scene, the hero revealed his deep psychological, so that the viewer a sense of kinship and realism ${ }^{[5]}$.

Museum space plot arrangement based on themes borrowed from literary narrative to arrange the order of the story space. Museum space plot layout object is to create a space orderly appeal by the museum interior space and they carry the story as a whole to consider, to cause a chain reaction and feelings in the viewer space experience, the experience of increased participation and fun, enhance the quality of museum space, making more space context, a sense of history, thus improving the social educational function of the museum, so that it can better and faster sustainable development. Overall design museum space plot is based on the theme of the story choreographed spatial structural integrity and design scenarios holistic design consultation to jointly release their story, so that the museum space to form a continuous surface with a comic book plot, the first echo, there are ups and downs of opening and closing, priorities, and then form can arouse the viewer's subjective feelings, wonderful story space. Fig.2 shows the folk custom exhibition hall.

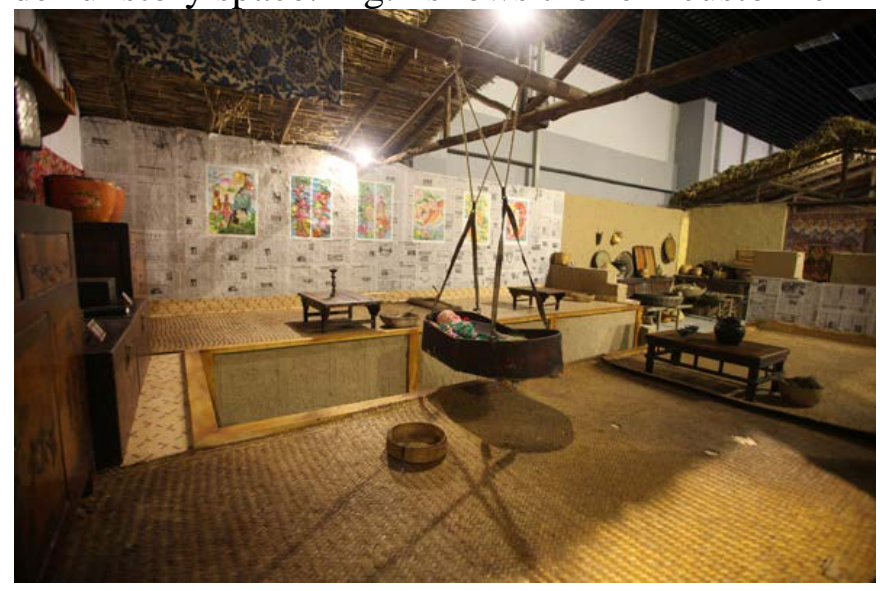

Fig. 2 The folk custom exhibition hall

As well as narrative theory, the museum's exhibition build two kinds of time dimension, time is a background exhibition itself exhibits show the content, a viewer is in the museum space by the designers involved in the exhibition to the time available. By this time the designer dimension 
displayed in the museum exhibits and reality together, is not part of the viewer experience the sense of the reality of that era. In this time, the designer of the show with the museum space is the order of the story appears, rather than the time actually happened. The selection and use of museum space, but also according to the inherent structure of the viewer is set, the architecture of this time based on the principles of formal narrative design logic in a more open, so that the viewer feel to visit significance and museum space to convey the message content. Personalized interior design emphasizes: Big industrial production to the community left the same problem stereotyped. Interior designers in the design process to be more emphasis on "man" is subject to the satisfaction of consumers, for the purpose of convenience. New materials are widely used: With the rapid development of new building materials, interior decoration materials, the future home will be fantastic.

\section{Conclusions}

Interior design is not only a matter of production, but also has the characteristics of social, cultural and art, it is these qualities make the interior gained more and more unpredictable significance. By interior design space chart, we can see that the interior design changes, and the impact of events in space. Comparison between the lateral interior design resources through the narrative, we can see that the narrative features, and its diversity, pluralism, difference and humanities, historical heritage. In this paper, the theory of narrative literature based research methods of spatial design, to explore how to use literary narrative way in interior design, spatial arrangement narrative content; content to explore how the integrity of the design space of the museum narrative; thus creating space contagious infection viewer, the viewer deepen understanding and memory, and in order to improve and perfect the social educational function of the museum, to make it better and faster sustainable development.

\section{References}

[1] Adrian Forty, Words and Buildings: A Vocabulary of Modern Architecture, London: Thames \& Hudson. 2002.

[2] Winfried Brenne. Practical Experience with the Buildings of the Avant-garde in Berlin and East Germany. The Soviet Heritage and European Modernism (International Council on Monuments and Sites). 2007

[3] Annemarie Jaeggi. Relations between the Bauhaus and the Russian Avant-garde as Documented in the Collection of the Bauhaus Archive Berlin. The Soviet Heritage and European Modernism (International Councilon Monuments and Sites), 2007.

[4] Sophia Psarra. Architecture and Narrative-The Formation of Space and Cultural Meaning in Buildings [M/CD]. New York: Routledge, 22009.

[5] Nigel Coates. Narrative Architecture [M /CD]. West Sussex: John Wiley \& Sons Ltd. 2012. 